\title{
Comparing memory capacity across stimuli requires maximally dissimilar foils: Using deep convolutional neural networks to understand visual working memory capacity for real-world objects
}

\author{
Timothy F. Brady \\ Department of Psychology \\ University of California, San \\ Diego
}

\author{
Viola S. Störmer \\ Department of Psychological and Brain \\ Sciences, Dartmouth College
}

Please address correspondence to:

Timothy Brady

9500 Gilman Dr. \#0109

McGill Hall 5322

La Jolla, CA, 92093

timbrady@ucsd.edu 


\section{Abstract}

The capacity of visual working and visual long-term memory play a critical role in theories of cognitive architecture and the relationship between memory and other cognitive systems. Here, we argue that before asking the question of how capacity varies across different stimuli or what the upper bound of capacity is for a given memory system, it is necessary to establish a methodology that allows a fair comparison between distinct stimulus sets and conditions. One of the most important factors determining performance in a memory task is target/foil dissimilarity. We argue that only by maximizing the dissimilarity of the target and foil in each stimulus set can we provide a fair basis for memory comparisons between stimuli. In the current work we introduce a new way to pick such foils objectively for complex, meaningful real-world objects by using deep convolutional neural networks, and we validate this using both memory tests and similarity metrics. Using this method, we then provide evidence that there is a greater capacity for real-world objects relative to simple colors in visual working memory; critically, we also show that this difference can be reduced or eliminated when non-comparable foils are used, potentially explaining why previous work has not always found such a difference. Our study thus demonstrates that working memory capacity is not fixed capacity but depends critically on the type of information that is remembered, and offers a solution of how to compare memory performance and other cognitive systems across different stimulus sets on common ground.

Keywords: memory capacity; visual working memory; similarity; deep learning; convolutional neural networks 


\section{Introduction}

One major focus of memory research is to examine, and quantify, how much information we can remember. For example, studies of visual long-term memory have shown that people can remember massive amounts of visual information (Standing, 1973; Brady, Konkle, Alvarez \& Oliva, 2008), and attempts have been made to quantify the upper bounds on his capacity (e.g., Landauer, 1986) in order to understand how memory might limit or interact with other cognitive systems (e.g., object recognition; Palmeri \& Tarr, 2008).

The domain of memory where capacity has been seen as most relevant, however, is visual working memory. In contrast to long-term memory, visual working memory is used to hold visual information actively in mind for relatively short periods of time and has a stark capacity limit (Baddeley, 2012; Cowan, 2001). Importantly, individual differences in this capacity limit are closely related to differences in fluid intelligence and academic achievement (Alloway \& Alloway, 2010; Fukuda et al., 2010), which is one of the reasons why describing and understanding these limits is of broad general interest. Theories markedly differ about the nature of the capacity limits of this system (e.g., Luck \& Vogel, 2013; Ma, Bays, Husain, 2013; Schurgin et al. 2020).

One particularly important question distinguishing theories of working memory limits has been whether the stimulus itself matters for working memory capacity: that is, does working memory capacity differ fundamentally for different stimuli, or is there a single fixed visual working memory capacity regardless of what we are remembering? Many studies have asked this question, comparing, for example, working memory for simple unidimensional stimuli (e.g., colors, orientations) to more complex meaningless stimuli (Alvarez \& Cavanagh, 2004; Awh et al., 2007) or to realistic objects (Brady et al., 2016), and making claims about the fundamental 
nature of working memory capacity as a result (e.g., fixed numbers of objects or not; fixed capacity or not). In the current work we focus on one particularly critical aspect of making such comparisons - the similarity of foil or lure items to the remembered item during the memory test - and how this seemingly simple aspect of such studies can fundamentally change our inferences about the underlying nature of visual working memory.

Standard assessments of visual working memory - particularly for stimuli that cannot make use of continuous adjustment tasks - ask participants to remember several items and then, at test, to distinguish whether a given item is old or new ('change detection', where 'new' items are the relevant 'lure'), or which of two items has been previously seen ('2-AFC', where the 'new' item is the 'foil'). In either case, the similarity of the lure or foil item is critical to performance (e.g., Awh et al. 2007; Schurgin et al, 2020). For example, if remembering the color red, then performance will be higher when your memory is tested against a blue lure than if you are asked to distinguish between the red item you saw and an orange lure item (e.g., Schurgin et al., 2020).

In the case of color and other simple low-level stimuli often used in visual working memory tasks, foils that are maximally distinct are commonly used - at minimum, large cross-category color differences (e.g., Luck \& Vogel, 1997). In many cases where continuous color spaces are used (e.g., Wilken \& Ma, 2004), foils are even more distinct: for example, when probing memory with a 2-alternative forced-choice, many studies use colors which are 180 degrees away on the chosen color circle (Li et al. 2020; Quirk et al. 2020; Brady et al. 2016), very close to as far as possible apart in that feature space given the color circles are generally chosen to be approximately maximally large for a given luminance level (Zhang \& Luck, 2008). This decision is, it turns out, critical: maximally distinct foils from within the feature space are the only nonarbitrary way to measure performance in a feature space, since performance can be driven lower from that point but never higher. If participants were asked to distinguish two extremely 
similar colors at test (e.g., red vs orange), performance would necessarily be lower, relative to when they are asked to distinguish between very distinct colors (e.g., red vs. blue); but within a given luminance level, performance can never be higher than when the foil is maximally far apart from the target color in hue. For color, then, performance on these commonly used tasks with maximally dissimilar foils is interpretable as approximately the upper bound of memory performance for that particular feature space, and thus as a valid measure of the 'capacity' of the memory system for colors.

By contrast, there is considerably more variability in the way foils have been selected when assessing both long-term memory capacity (e.g., Brady et al. 2008) and working memory capacity for complex objects (e.g., Alvarez \& Cavanagh, 2004 vs. Awh et al. 2007) and realistic, meaningful objects (e.g., Brady et al. 2016 vs. Li et al. 2020), and this has significant potential implications for conclusions about the nature of memory capacity. As is the case for colors, memory performance is necessarily lower when participants are asked to make comparisons between more similar objects at test: for example, people perform worse with within-category foils (Brady et al. 2016; Brady et al. 2009) and the similarity of foils impacts performance for both realistic objects and complex but meaningless objects (Frank et al. 2020; Mate \& Baqués, 2009). Thus, as is the case for color, when trying to assess overall memory capacity for complex or realistic objects, it is necessary to choose maximally distinct foils, or else performance will be arbitrarily lower — and the more similar the foils, the lower performance will be. However, these performance declines are only due to perceptual or conceptual confusability of items with the foils, and do not reflect a true decline in memory capacity. The fact that maximally distinct foils have not always been used is thus critical to a wide variety of literatures that assess memory performance and make inferences about the nature of capacity limits. 
One example of this from visual long-term memory is work by Brady et al. (2008) that sought to assess and quantify visual long-term memory capacity. Although aiming to measure the upper bound of memory performance, Brady et al. (2008) did not use maximally dissimilar foils, but instead randomly chosen foils from their stimulus set. Thus, despite the impressive performance they found, to the extent that randomly chosen foils are not maximally dissimilar, they likely underestimated the true performance level that can be achieved in visual long-term memory.

In the current work, we focus on one particular example of this general issue: the case of comparing working memory capacity for objects to other simple stimuli, like colors. Comparing memory performance across different stimuli is a case where the strategy used to choose foils is absolutely critical, because foils are necessarily different for different kinds of stimuli, which means comparisons must always depend on the particular foils used for each stimulus set. In the case of meaningful objects vs. colors in particular, some work has found significant working memory performance advantages for real objects when compared to simple stimuli like colors (Brady et al. 2016; Brady \& Störmer, 2020), where other work claiming to be a direct replication of Brady et al. (2016) has not (e.g., Quirk et al. 2020; Li et al. 2020). This has led to different assessments of the very nature of working memory storage, and in particular the impact of knowledge and familiarity on memory capacity and whether it makes sense to suppose there is a fixed working memory capacity at all.

However, there is a critical difference in these studies: in all of these experiments, the color foils were chosen to be maximally distinct in CIE $L^{*} a^{*} b$ space, as is typical. However, Brady et al. (2016) also chose the real-world object foils to be of different categories as well as perceptually 
and conceptually dissimilar ${ }^{1}$. However, later follow-up studies that found similar capacities between meaningful objects and colors did not do this, instead randomly choosing foils from a stimulus set of items from different categories (Quirk et al, 2020; Li et al. 2020). That is, despite claiming to be exact replications of Brady et al. (2016), these studies selected their foils differently than the original study of Brady et al. (2016). Instead of attempting to maximize the dissimilarity of objects both categorically and visually within the Brady et al. (2008) stimulus set, as Brady et al. (2016) had, these studies chose foil objects randomly from this large set of cross-category objects, and did not ensure that foils were visually or conceptually distinct from the target - meaning that a significant percentage of the time, the object foil was visually and even semantically similar to the target item. That is because in the Brady et al. (2008) stimulus set, which has thousands of images from distinct basic-level category images, there are still significant gradations of how similar the objects are semantically and visually (e.g., an office chair and a dining room chair are both included, and while they are dubbed distinct categories in the stimulus set, it is clear that they are similar; likewise, a tape measure and a ruler are both included; etc) - so choosing foils randomly from other categories than the target stimulus does not, in fact, maximize semantic or visual distinctiveness.

In the current work, we seek to understand the role of foil similarity decisions in assessments of memory capacity, and to test a new way of measuring foil similarity for realistic objects in a way that predicts memory performance. In particular, we quantify the foil similarity that arises from choosing objects randomly from stimulus sets like that of Brady et al. (2008); show this has major implications for visual working memory performance; and demonstrate that the difference in behavioral results between studies that compare objects and colors in visual working memory

\footnotetext{
${ }^{1}$ Brady et al. (2016) specifically reported they performed "a 2AFC memory test with either objects or colors that were as distinct as possible from the encoded objects/colors"; in practice, we simply generated the pairs in advance and eliminated any pairs that were visually or conceptually similar.
} 
and arrive at different answers (e.g., Brady et al. 2016 vs. Li et al. 2020) can be explained — at least in large part - by this difference in foil similarity. In particular, in the current work we show how working memory performance differs for both colors and real-world objects depending on whether the foils are chosen to be maximally dissimilar or not.

While creating maximally distinct foils is fairly straightforward for colors and other simple lowlevel features - because we can relatively directly measure perceptual distinctiveness, for example as distance in CIE $L^{*} a^{*} b$ space for color - it is not obvious what a maximally distinct foil should be for a real-world object. To operationalize this, we introduce a novel method to formally assess real-world object similarity using a deep convolutional neural network (CNN) that allows us to objectively identify distinct foils, similar to how foils for simple visual stimuli like color are chosen. In particular, we use the VGG16 pre-trained convolutional neural network to assess image similarity between each pair of objects from the thousands of objects in the Brady et al. (2008) stimulus set. This convolutional neural network is trained to go from images (pixels) to category labels (e.g., ball) and, in the past 10 years, such networks have become increasingly good not only at solving object recognition from images, but also increasingly good as models of visual processing in the primate visual system (e.g., Yamins, et al. 2014). Thus, they provide a useful way to find approximately maximally dissimilar object images without requiring participants to give similarity ratings on millions of pairwise object sets. At the same time, using such networks provides additional information about the networks as models of human information processing: to the extent VGG16-chosen foils are indeed more distinct than random foils, this suggests convolutional networks of this type provide a useful model of human memory confusability.

Overall, in a series of high-powered, preregistered experiments, we find that (1) foils chosen to be dissimilar by VGG16 are, in fact, particularly dissimilar according to human participants; (2) 
this dissimilarity modulates memory performance, such that participants perform better at memory tasks with such dissimilar foils than the randomly-chosen cross-category foils sometimes used in previous work, and (3) when we use equivalently chosen, maximally dissimilar foils for both colors and objects, we find a large benefit for objects relative to colors at long encoding times, replicating previous results of higher working memory capacity for

meaningful objects (Brady et al., 2016; Brady \& Störmer, 2020; Asp et al., 2019). Consistent with this as an explanation for previous failures to find such advantages, we also find such effects are reduced when comparing maximally distinct color foils vs. randomly-chosen, nonmaximal object foils, indicating that the lack of behavioral object benefits in some previous studies (Quirk et al., 2020; Li et al., 2020) were indeed due to their making an unfair comparison between different kinds of stimuli. Broadly, our results demonstrate that realistic objects result in better working memory performance than simple features once foil dissimilarity is matched, emphasizing the importance of comparing different sets of stimuli on common ground, a general challenge in many studies trying to compare cognitive operations across different stimulus sets; and provide a means of generating such foils objectively and automatically.

\section{Experiment 1: Stimulus creation and validation}

For objects, we used the Brady et al. (2008) object image database, as in the Brady et al. (2016), Quirk et al. (2020) and Li et al. (2020) studies of visual working memory capacity for objects. This database contains 2400 objects, with, as best as possible, one object per 'basiclevel category' (e.g., one office chair). In our first experiment, we ask whether, within this database, there is variability in how similar randomly chosen pairs are, despite the objects being cross-category. In subsequent experiments we ask whether this variability is critical to explaining differences in visual working memory performance. Experiment 1 thus measures the similarity of different pairs of objects within the database, using both convolutional neural 
networks and human similarity ratings, as a precursor to the memory experiments (Experiments 2 and 3).

With 2400 distinct objects there are potentially greater than 5 million possible pairs of stimuli that could serve as the studied item/foil. Thus, finding the maximally dissimilar pairs from this set is not straightforward. To address this, we make use of deep convolutional neural networks to achieve this goal. Recently deep convolutional neural networks have reached very high performance on object recognition tasks, reaching, and even surpassing in limited circumstances, human category-level recognition performance (He et al., 2016; Kietzmann et al., 2018; Lindsay, 2020; Russakovsky et al., 2015; Yamins \& DiCarlo, 2016). Furthermore, evidence suggests that these networks capture some aspects of the neural representations of visual information, as they have been shown to account for neural data recorded in higher-level visual areas of the human and non-human primate brain (Kubilius et al. 2018, Khaligh-Razavi \& Kriegeskorte, 2014; Yamins et al., 2014; Güçlü \& van Gerven, 2015; Kar et al., 2019). While there is still some debate of how well CNNs capture human vision (e.g., Xu \& Vaziri-Pashkam, 2019), and different model architectures result in different performance levels and how well they explain neural representations (e.g., Storrs et al., 2020), overall it is clear that they provide one of the best current computational tools to model human object recognition, and, of most importance to the current paper, are sensitive to visual and category-based similarity of objects (Peterson et al. 2018). Thus, they provide a useful tool for choosing object pairs that will provide a fair assessment of the upper bound on memory performance, allowing us to measure memory capacity in a fair way. 


\section{Method}

\section{Creating pairs}

To choose maximally dissimilar and maximally similar foils from within the Brady et al. (2008) database and see how they compare to randomly selected foils, we used a VGG16 convolutional neural network (CNN) architecture (Simonyan \& Zisserman, 2014), pretrained on ImageNet, to select images based on image-level similarity. This model is a deep convolutional neural network with 16 layers, and among the most well-known and well-cited models of its kind. It achieves $92.7 \%$ top- 5 test accuracy (i.e., the correct label was in the top 5 suggestions by the network) on ImageNet, a dataset of over 14 million images.

Using this model allowed us to create 'maximally dissimilar' and 'maximally similar' object foils, at least with respect to the features of this network. To do so, we calculated the features of all of our object images using the CNN for all objects in our database, using the Keras implementation of ImageNet-pretrained VGG16 in Tensorflow. Then we used the CNN feature matrix from the top max-pooling layer (with images 256x256, 8x8x512 =32,768 features/image) to compute similarity between all pairs of objects (cosine similarity; i.e., length-normalized dot product), and chose both the 120 most dissimilar and most similar pairs with only the constraint that no object appeared in multiple pairs. Creating the maximally similar pairs revealed that one image was duplicated in the Brady et al. (2008) set, and so we replaced this image. We used the top max-pooling layer as we were most interested in the features that are used for categorization/classification, as opposed to the lower layers that are more similar to earlier visual regions (e.g., Yamins et al. 2014).

Note that despite being a model that works on image features, such networks are trained to do categorization, and so even though we use the network for feature extraction rather than categorization, the top layers of such models contain category-specific information, designed to 
be read-out by the fully-connected categorization layers that are omitted when doing feature extraction. Thus, such deep nets trained on categorization are sensitive to some extent to both visual and semantic features (e.g., Jozwik et al. 2017; Peterson et al. 2018). Given that such deep convolutional neural networks are useful models of human recognition and the human visual system (e.g., Yamins, et al. 2014), these pairs should be more dissimilar than randomly chosen pairs, although they may not be as dissimilar as pairs chosen specifically to avoid similarity by humans (as used in Brady et al. 2016). We have publically shared the code to extract features for the objects:

https://colab.research.google.com/drive/1vKpxABn0J9vi vPleBb-XQdrlQmhgc4L 

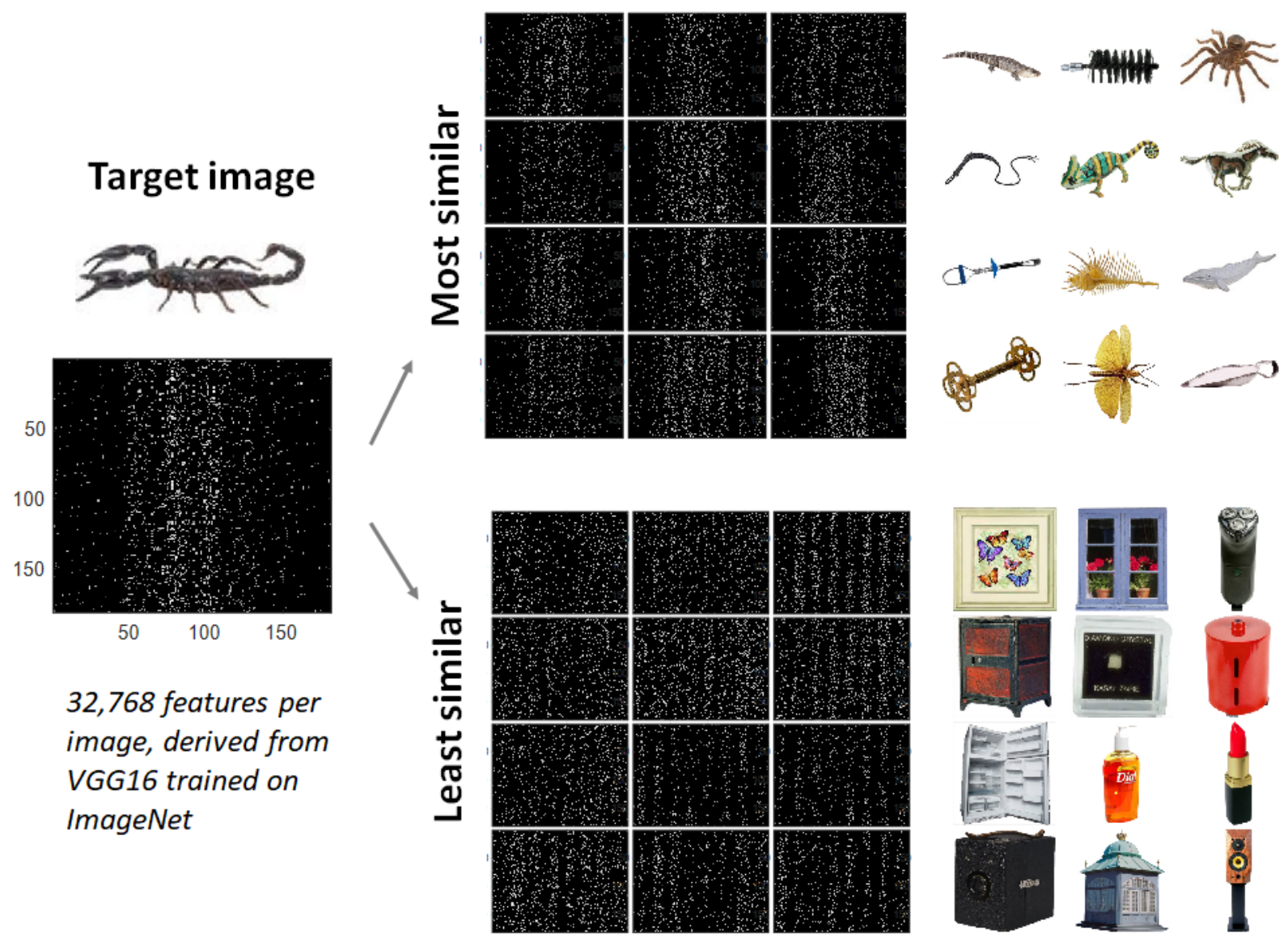

Figure 1. Designing maximally-distinct foils using a deep convolutional neural network to measure similarity. From each image, we extracted features from VGG16, a network trained to do object classification on ImageNet. The features for each object are visualized in the black/white plots, which show white for more activity for a given feature and black for less. We used these features to select, for each image, the most dissimilar foil. Here, we visualize the most similar and most dissimilar items for one particular target image. Deep neural networks are trained on categorization, and thus are sensitive to both visual and semantic features. For example, note that the most similar items to the example target image are largely animals, with similar shape and texture; whereas the least similar items are inanimate, with different shapes and textures. 


\section{Maximally similar cross- category pairs}

Random crosscategory pairs

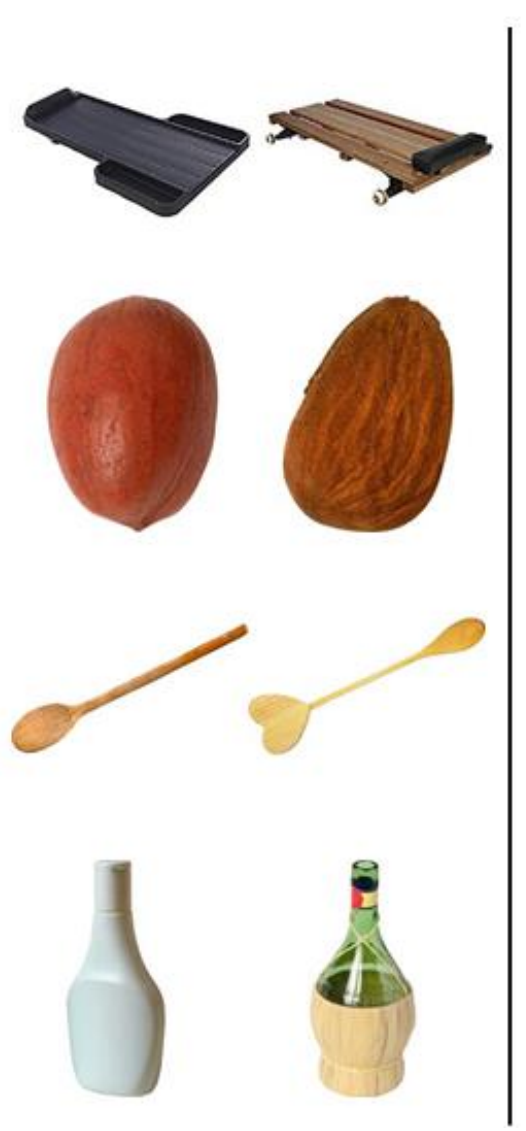

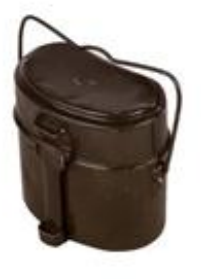
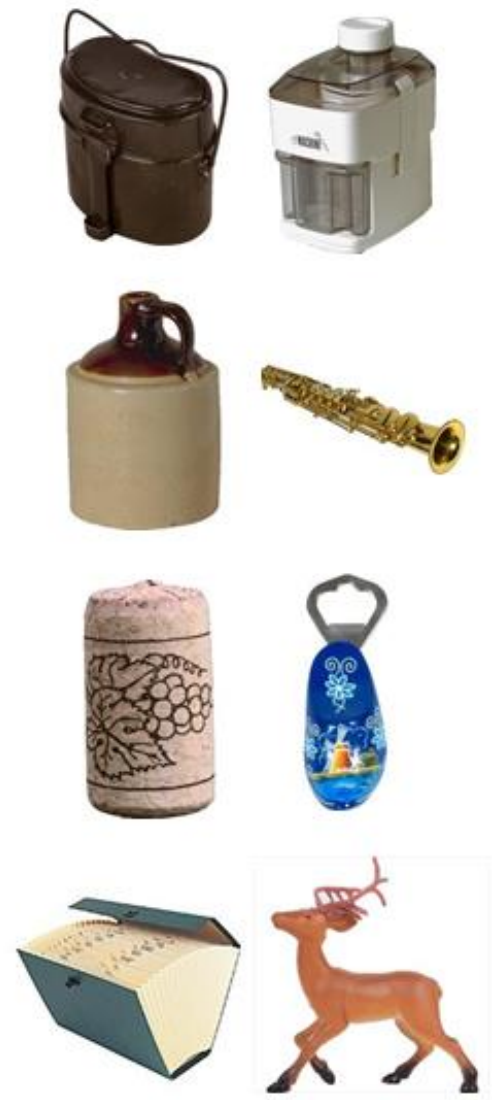

\author{
Maximally \\ distinct cross- \\ category pairs
}

Figure 2. Representative pairs of objects that were derived from the convolutional neural net and that served as the study item and foil items in the memory experiments (i.e., one item from each pair would be studied and the other would serve as the foil at test for that item). The 'maximally similar' and 'maximally distinct' pairs chosen by the convolutional neural network features appear to be more similar, or more divergent, both semantically and visually than randomly chosen pairs despite all the pairs being 'cross category'. Also noteworthy is that despite being cross-category, there exist pairs in the dataset that are incredibly similar, both conceptually and visually.

\section{Validation of pairs with another convolutional neural network}

As our goal is not to fully assess convolutional neural nets as models of human behavior and neural processing (e.g., Storrs et al. 2020). We simply wish to use such convolutional nets as a tool to choose dissimilar foils. So we compared the similarity of VGG16-chosen-foils to only one 
other convolutional net - helping ensure the conv. net similarities were not totally idiosyncratic to VGG16. In particular, we picked a distinct architecture, ResNet (He et al., 2015), and used ResNet-18 via the PyTorch implementation (Paszke et al., 2017), again focusing on the final max pooling layer. We assessed the similarity (again using cosine similarity) of all the pairs chosen by VGG16 in this network.

\section{Validation of pairs with human similarity data}

Participants. All experiments were approved by the Institutional Review Board at UC San Diego. Fifty US-based participants (33 male, 16 female, 1 other/chose not to say) from Amazon's Mechanical Turk were recruited to perform a similarity task (all with >=95\% previously accepted HITs).

Stimuli and Procedure: Participants completed 120 trials overall, 40 each (chosen randomly) from the set of 120 available in each of the three conditions (maximally-distinct cross-category object-foils; random cross-category object-foils; maximally-similar cross-category object foils). On each trial, participants were shown an object and asked "How similar are these objects?" on a scale from 1 (not similar) to 7 (maximally similar). We told participants we were interested in their intuitive visual judgments, and not the similarity of the words you might use to describe the objects, but otherwise left the task open-ended. Trials were not speeded and participants responded with the keyboard buttons $1-7$. When participants responded, the pair of objects disappeared, followed by a $500 \mathrm{~ms}$ delay and then the next pair of objects. Pairs from all conditions were interleaved randomly. 

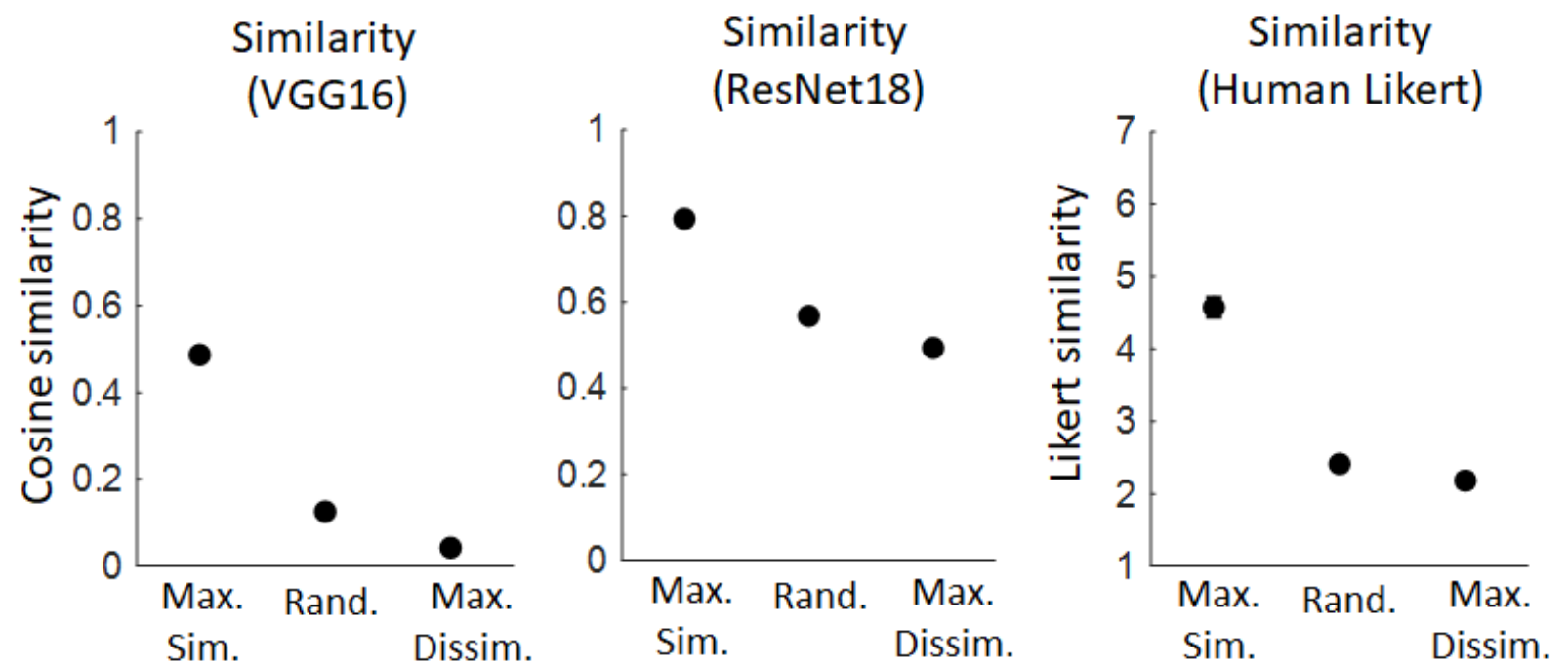

Figure 3. Similarity of each set of pairs, from both two different deep convolutional neural networks and from human similarity ratings. The similarity measures indicate that the VGG16 network effectively chose maximally similar and maximally dissimilar pairs, which differed from randomly chosen pairs in the predicted directions. Error bars are present on all graphs (by pair for the networks; by participant for the human similarity) but are generally smaller than the dots themselves.

\section{Results}

VGG16, naturally, predicts extremely low similarity for maximally dissimilar pairs, and quite high for maximally similar, as this is the network that was used to select the pairs and so these similarities are not independent of the condition they are in. Note, however, that in the maximally similar condition, similarities are nowhere near 1, as all of the images available in the Brady et al. 2008 dataset are distinct and cross-category.

The similarity of the two deep convolutional neural networks was extremely strongly related. Their measured similarities as a function of stimulus kind (VGG16-generated pairs and randomly paired objects) are plotted in Figure 3. ResNet-18 predicts nearly the same pattern as VGG16, despite not being used to select the stimuli, although there is an overall shift where all stimuli pairs are more similar in ResNet-18 than in VGG16. Across all 360 pairs, similarity 
derived from VGG16 and ResNet-18 had a correlation of $r=0.93(p<0.0001)$, suggesting both tap extremely similar features of real-world objects for the purpose of constructing such pairs.

Human likert ratings agree that in aggregate, the maximally similar pairs chosen by VGG16 are more similar than randomly paired stimuli $\left(t(49)=10.87, p<0.001, d_{z}=1.54\right)$; and maximally dissimilar pairs are less similar than randomly paired stimuli $(t(49)=-4.90, p<0.001)$. Note that this later effect was small in absolute terms but highly reliable $\left(d_{z}=0.69\right)$, in part because many participants gave all object pairs that were clearly of a different category the lowest similarity level.

How well do the networks capture human similarity? Both captured effectively all of the explainable variance in human similarity ratings for this set of 360 pairs. We took 1000 random split half correlations from the human similarity data and corrected them using the SpearmanBrown formula to get an estimate of the reliability of the human ratings for each of the 360 pairs. This gives a noise ceiling (e.g., a maximum expected correlation given the measurement noise of the human data per pair) of approximately $r=0.71$. The similarity ratings derived from ResNet18 correlate with the human data with $r=0.76(p<0.0001)$ and the similarity ratings derived from VGG16 correlate with the human data with $r=0.75(p<0.00001)$ - at the noise ceiling of the human similarity measure.

Overall then, stimulus selection and validation reveals that both VGG16 and ResNet-18 provide extremely good predictive power for choosing both minimally and maximally similar pairs for object images; and that, as expected, randomly chosen pairs from the Brady et al. (2008) stimulus set are not maximally dissimilar, making them not a valid measure of memory performance for comparing across stimulus sets (e.g., for comparing with maximally dissimilar color foils). 


\section{Experiment 2: Object foil similarity determines performance even within a set of cross- category objects}

Experiment 1 reveals that within the stimulus set used by much prior work on visual working memory and visual long-term memory (Brady et al. 2008), randomly chosen pairs of objects, despite being putatively cross-category, are not maximally-dissimilar. Does this have implications for memory performance? Or does any foil that is reasonably distinct from the target object give the same memory performance?

To assess this, in Experiment 2 we asked what the role of such similarity differences is for visual memory performance. In particular, we had participants remember real-world objects in visual working memory, and then at test, had them do a 2-alternative forced-choice memory test. The study-test pairs in the 2-AFC were drawn from either the maximally-similar, randomly-paired, or maximally-dissimilar stimulus pairs developed in Experiment 1. If randomly-chosen foils are sufficiently dissimilar to provide a fair measure of the upper bound on memory performance (e.g., as assumed by Brady et al. 2008; Quirk et al. 2020; and Li et al. 2020) there should be no difference between randomly-chosen pairs and maximally-dissimilar pairs in memory performance. By contrast, if memory performance is significantly impacted by similarity even for maximally-dissimilar vs. random pairs, then this would suggest that memory is underestimated for real-world objects when using randomly-chosen foil pairs, and cannot be fairly compared to memory for other stimuli (e.g., colors) which use maximally-dissimilar pairs. 


\section{Methods}

The study design, hypothesis, analysis plan and exclusion criteria were preregistered: https://aspredicted.org/blind.php?x=c9nd9e. Materials, data and analysis code are available at https://osf.io/axyqs/

Participants: Fifty US-based participants (34 male, 16 female, 0 other/chose not to say) from Amazon's Mechanical Turk were included in the final data set (all with >=95\% previously accepted HITs). 11 additional participants were excluded and replaced based on our preregistered exclusion criteria.

Stimuli and Procedure: We used the Brady et al. (2008) object image database, as described above. In particular, in the random-pairs condition, we choose each pair of objects randomly from the full set of 2400 objects without regard to similarity. For the maximally similar and dissimilar cross-category foils, we used the sets generated as described in Experiment 1: all the images were from the set of 2400 cross-category objects, but chosen in such a way as to maximize or minimize similarity.

Participants completed 180 trials overall, 60 in each of the three conditions (maximally-distinct cross-category object-foils; random cross-category object-foils; maximally-similar cross-category object foils). On each trial, participants were asked to remember 6 objects that were shown for $2,000 \mathrm{~ms}$. The long encoding time, combined with fixed spatial positions with placeholders present during the delay, helped ensure there was little to no location noise that can cause misbinding. After a delay period of $700 \mathrm{~ms}$, one of the items was probed in a 2-alternative forced choice (2AFC) format. One location was cued and two stimuli - one that was previously seen on the memory display and one foil — were shown in the center of the screen and participants 
had to indicate which of these two items (left vs. right) was part of the initial memory display (Figure 4). All trial types were randomly interleaved.

Verbal overshadowing was performed while encoding and memorizing the objects. In other studies we have shown that in-lab verbal interference (that is monitored continuously by an experimenter) and mental rehearsal of a single word during online studies results in similar visual working memory performance (Brady \& Störmer, 2020). Thus, participants were instructed to mentally rehearse the word 'the' for the entire duration of the encoding period. They were reminded of this on every trial, as well as the need to use purely visual memory and not use words to remember the stimuli.

Analysis. Working memory performance was quantified using d' for a 2-AFC task, $[z H-z F A] / \sqrt{ } 2$. Where $P$ is percent correct and $\Phi$ is the Gaussian cumulative distribution, $z H=\Phi(P)$ and $z F A=$ $\Phi(1-P)$. Per the preregistration, data were excluded if performance averaged across all conditions was below 0.5 , or if greater than $10 \%$ of individual trials were excluded. Individual trials were excluded if: 1) A response occurred less than $150 \mathrm{~ms}$ after the response screen appeared. 2) The response occurred more than $5 \mathrm{~s}$ after the response screen appeared. 


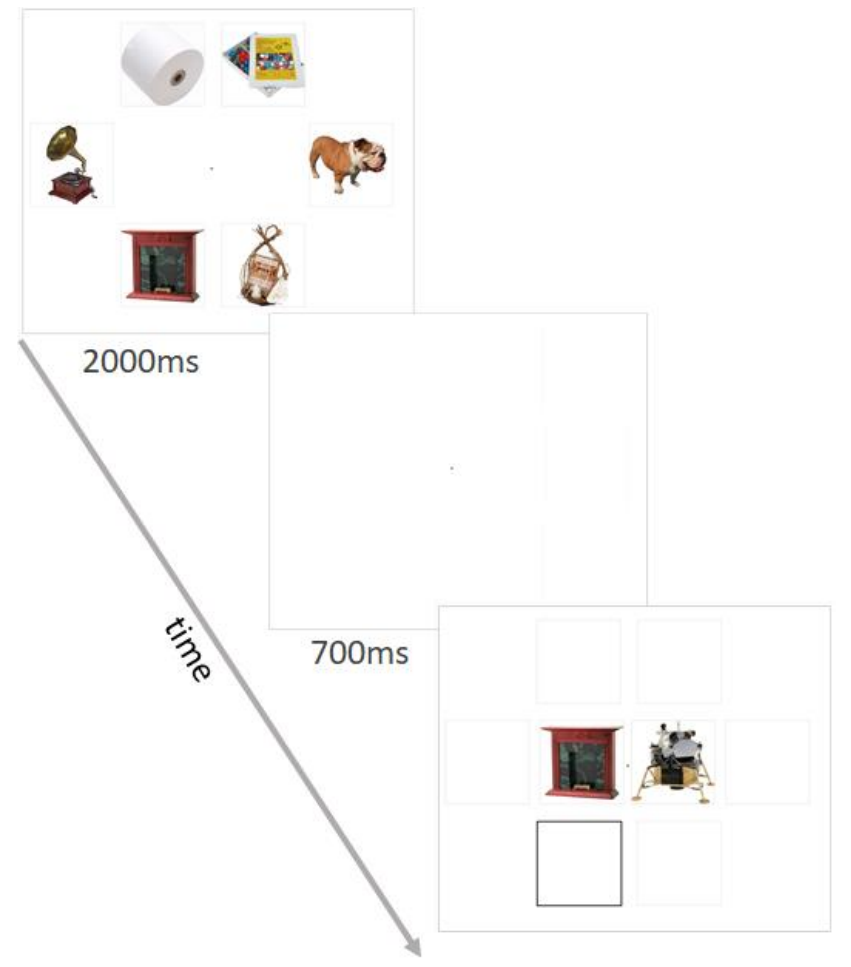

Figure 4. Method of Experiment 2. Participants saw 6 objects, and remembered them over a short delay. They were then presented a 2-AFC probe and needed to choose which item was presented at the cued location. The foil items in the 2-AFC memory test could be either maximally-distinct cross-category foils, minimally-distinct cross-category foils, or randomlychosen from a different category. 


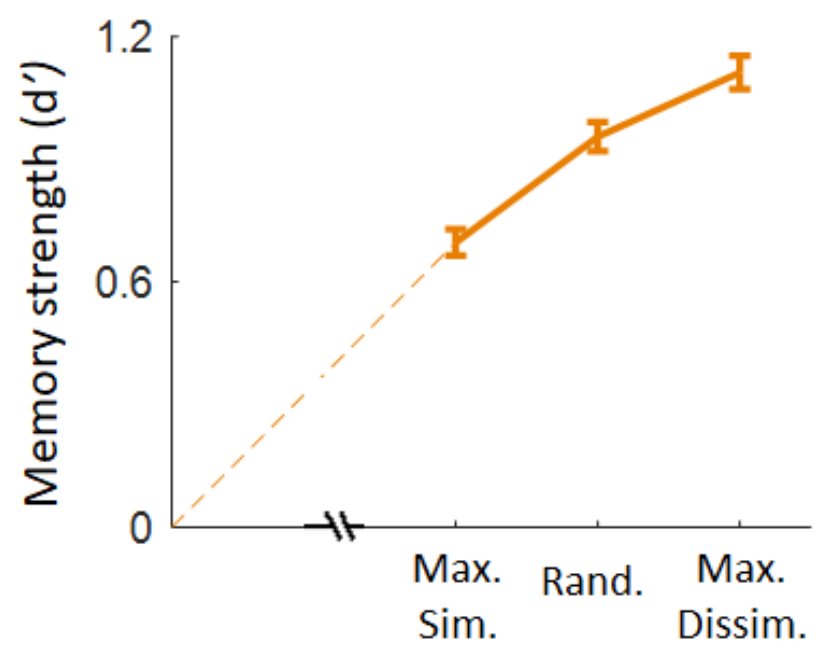

\section{Foil dissimilarity $\rightarrow$}

Figure 5. Results of Experiment 2. We find that randomly chosen pairs are not in fact maximally dissimilar for the purposes of memory: participants perform better when the foils are maximally distinct than when they are randomly selected. They perform worst when foils are maximally similar within this across-category stimulus set. We also visualize how performance must necessarily increase as foils become more dissimilar (dotted line); for example, with nearidentical completely similar foils (e.g., a 1 pixel difference between target and foil), performance would be at chance $\left(d^{\prime}=0\right)$. This means only maximally-dissimilar foils are non-arbitrary ways of measuring memory performance, providing the upper bound of memory strength.

\section{Results}

Participants overall showed higher memory performance for more distinct foils, even though all foils were cross-category foils (Figure 5). An analysis of variance (ANOVA) with foil type (maximally similar, random, maximally distinct) as factors confirmed there was a main effect $(F(1,49)=22.18, p<0.0001)$. Planned follow-up pairwise comparisons showed that maximally similar cross-category object pairs resulted in lower performance than random cross-category pairs $\left(\mathrm{t}(49)=4.79, \mathrm{p}<0.001, \mathrm{~d}_{z}=0.68\right)$ and random cross-category pairs resulted in lower performance than maximally-dissimilar cross-category pairs $\left(t(49)=2.26, p=0.028, d_{z}=0.32\right)$. 
Note that human similarity ratings were similar for maximally dissimilar objects and randomly chosen objects, even if the difference between them was reliable; we noted this is in part because many participants gave all object pairs that were clearly of a different category the lowest similarity level. The memory data from this experiment - where the gain was considerable for maximally dissimilar foils relative to randomly chosen foils - suggests that this overall small difference in similarity rating likely obscures differences in their conceptual and perceptual relatedness that do, indeed, matter quite a bit for memory performance. This is perhaps what has driven some past researchers (including Brady et al. 2008, as well as Li et al. 2020 and Quirk et al. 2020) to treat cross-category foils as 'dissimilar enough' to judge memory capacity. Yet this Experiment suggests that these differences matter substantially for memory performance. Thus, different levels of what — for human judgments - seem to be effectively floor levels of similarity may matter for human memory performance, making the convolutional neural network approach to stimulus generation particularly well-suited for this stimuluschoosing task.

\section{Experiment 3: Objects are better remembered than colors with matched foil similarities}

We have shown that within the cross-category stimulus set of Brady et al. (2008) used in many previous working memory studies, there is room for significant variation in foil difficulty. Does this variation - and the failure of some previous studies to use maximally-distinct foils both in terms of visual and categorical similarity (e.g., Li et al. 2020; Quirk et al. 2020) explain why they did not find a benefit for real objects compared to colors in working memory? In Experiment 3, we examine that question directly, asking if this deviation from the way foils were chosen by Brady et al. (2016) is critical to the difference in results these studies obtained. To do so, we directly compare memory for objects to memory for colors at long encoding times (2sec), which 
is the condition where these studies diverge, with Brady et al. (2016) finding a significant benefit for objects at such long encoding times.

\section{Method}

The study design, hypothesis, analysis plan and exclusion criteria were preregistered: https://aspredicted.org/blind.php? $\mathrm{x}=\mathrm{s} 8 \mathrm{bd} 3 \mathrm{w}$. Materials, data and analysis code are available at https://osf.io/axyqs/

Participants: Fifty US-based participants (30 male, 19 female, 1 other/chose not to say) from Amazon's Mechanical Turk were included in the final data set (all with $>=95 \%$ previously accepted HITs). 9 additional participants were excluded and replaced based on our preregistered exclusion criteria.

Stimuli and Procedure: We contrasted randomly-chosen foils and maximally-dissimilar foils for both objects and colors in a standard long encoding time working memory task modeled after Brady et al. (2016). For objects, we used the maximally-dissimilar foils derived by VGG16 as described in Experiments 1 and 2, and for randomly-chosen foils we chose each pair of objects randomly from the full set of 2400 objects without regard to similarity, as described above and as done in recent papers (e.g., Li et al. 2020; Quirk et al., 2020). As in Experiment 2, one item from each pair was shown in a memory display, and the other served as the foil during the 2AFC memory test. Which item served as the memory item vs. foil in each pair was randomized across participants.

For colors, we used a standard color circle (Suchow et al. 2013; Schurgin et al. 2018) of radius 49 in the CIE $L^{*} a * b$ space (centered at $L=54, a=21.5, b=11.5$ ). We created two analogous 
conditions to the object conditions: the maximally distinct color pairs condition, with foils $180^{\circ}$ away from the target color (as is traditionally done); and a 'random' foil condition, where the foil color could be any color that was $>30^{\circ}$ from the target; e.g., foil colors ranging from $30-180$ degrees away, randomly chosen on each trial. We choose this limit to mimic the distinct object categories in the object set, as this limits foils to be different color categories (by carving out a wedge of $60^{\circ}$ of the color wheel around the target). To limit ensemble-based encoding (e.g., Brady \& Alvarez, 2015), we chose all of the memory colors and the foil color subject to the constraint that no two colors could be less than $15^{\circ}$ apart on the color wheel.

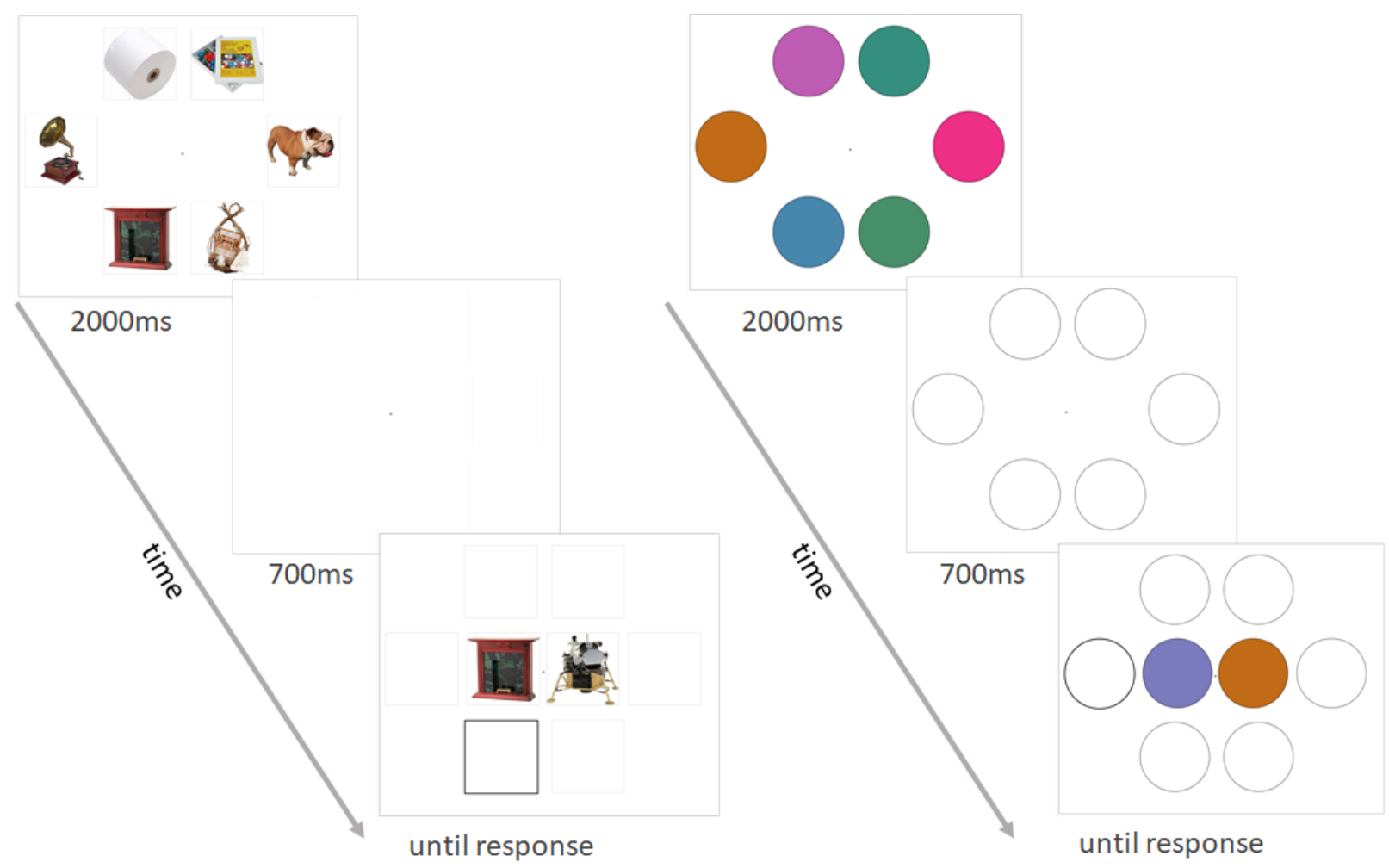

Figure 6. Method of Experiment 3. Participants saw 6 items, either objects or colors, and remembered them over a short delay. They were then presented a 2-AFC probe and needed to choose which item was presented at the cued location. The foil items in the 2-AFC memory test could be either maximally-distinct or randomly-chosen from a different category. 
Participants completed 160 trials overall, 40 in each of the four conditions (maximally-distinct object-foils; random object-foils; maximally-distinct color foils; 'random' color foils). On each trial, participants were asked to remember 6 stimuli - either colors or objects - that were shown for 2000ms. The long encoding time, combined with fixed spatial positions with placeholders present during the delay, helped ensure there was little to no location noise that can cause misbinding. After a delay period of $700 \mathrm{~ms}$, one of the items was probed in a 2 -alternative forced choice (2AFC) format. In particular, one location was cued and two stimuli — one that was previously seen on the memory display and one the pre-chosen foil - were shown in the center of the screen and participants had to indicate which of these two items (left vs. right) was part of the initial memory display (Figure 6). All trial types were blocked.

Verbal overshadowing was again performed while encoding and memorizing the objects, as participants were instructed to mentally rehearse the word 'the' for the entire duration of the encoding period. They were reminded of this on every trial, as well as the need to use purely visual memory and not use words to remember the stimuli.

Analysis. Just like in Experiment 2, working memory performance was quantified using d' for a 2-AFC task, $[z H-z F A] / \sqrt{ } 2$. Where $P$ is percent correct and $\Phi$ is the Gaussian cumulative distribution, $\mathrm{zH}=\Phi(\mathrm{P})$ and $\mathrm{zFA}=\Phi(1-\mathrm{P})$. Per the preregistration, data were excluded if performance averaged across all conditions was below 0.5 , or if greater than $10 \%$ of individual trials were excluded. Individual trials were excluded if: 1) A response occurred less than 150ms after the response screen appeared. 2) The response occurred more than $5 s$ after the response screen appeared. 


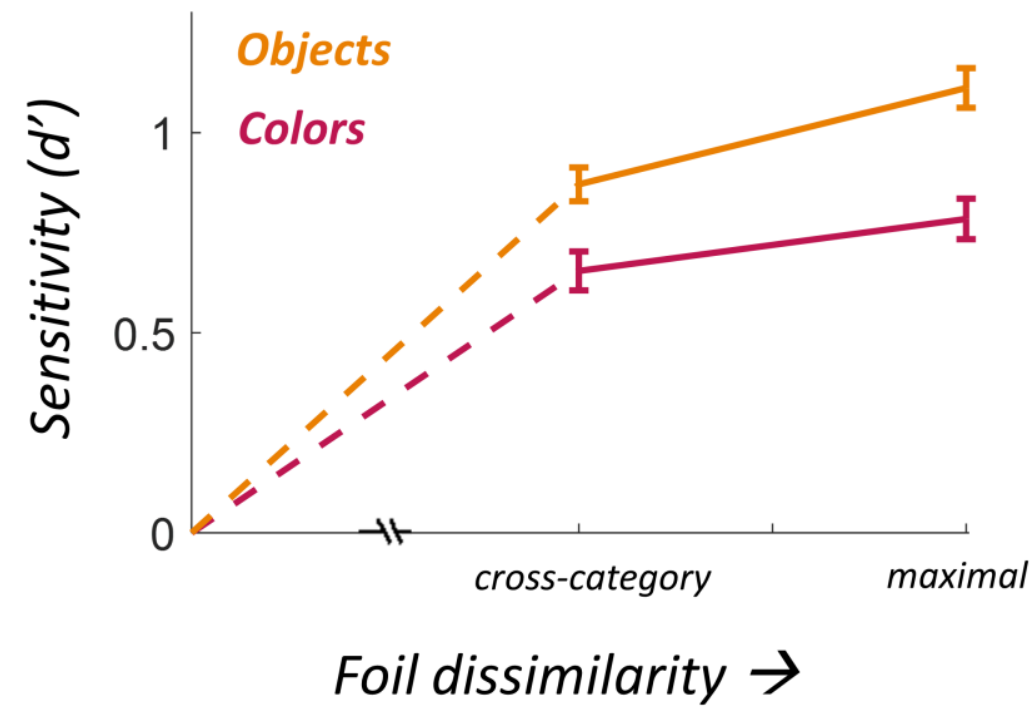

Figure 7. Results of Experiment 3. We find that while cross-category changes are indeed large changes - and thus result in well-above chance memory performance for both colors and objects - performance is best for both colors and real-world objects when foils are maximally distinct. Thus, to compare memory performance across stimulus sets in a fair way, the foils need to be chosen the same way. These data show that once test foils are matched in terms of their similarity to the target, there is a clear object benefit in working memory performance. Only when making the unfair comparison of cross-category object changes (chosen at random) vs. maximally dissimilar color changes is there no benefit for objects. As is the case for objects, it is also the case that for colors, with near-identical foils (e.g., a 1 deg. difference on the color wheel), performance must be at chance ( $\left.d^{\prime}=0\right)$, and performance must necessarily increase as foils become more dissimilar. This means that for both objects and colors, only maximallydissimilar foils are non-arbitrary ways of measuring memory performance.

\section{Results}

Participants overall showed higher memory performance for real-world objects relative to colors, as well as higher memory performance for maximally dissimilar foils relative to randomly picked foils for both colors and objects (Figure 7). An analysis of variance (ANOVA) with stimulus type (objects, colors) and foil type (distinct, random) as factors confirmed there were two main effects (objects $>$ colors; $F(1,49)=15.05, p=0.0003$; distinct $>$ random; $F(1,49)=14.932, p=0.0003$ ), and no interaction $(F(1,49)=1.56, p=0.22)$. Planned follow-up pairwise comparisons showed that that foil type affected performance for both objects $\left(t(49)=4.02, p<0.001, d_{z}=0.57\right)$ and to some extent for colors $\left(t(49)=1.84, p=0.071, d_{z}=0.26\right)$. 
Overall, these results replicate the object-advantage previously reported by Brady et al. (2016) and replicated by Brady and Störmer (2020), and demonstrate the importance of choosing comparable test foils across stimulus sets when assessing memory performance. When comparing conditions in which color foils are chosen to be maximally distinct from the target color, but objects are tested against object foils chosen at random - as done in recent papers (Quirk et al. 2020; Li et al.2020) — we find no reliable performance difference between colors and objects $\left(t(49)=1.06, p=0.296, d_{z}=0.15\right)$. Yet it is clear that this is only due to the unfair comparison, where color and object memory are not tested on even ground. Thus, the comparison used in these papers - between randomly-chosen objects and maximally distinct colors - cannot be interpreted as a comparison of memory performance between colors and real-world objects.

\section{General Discussion}

The present results demonstrate the importance of assessing memory capacity in a fair and consistent way across stimulus sets, and point to the significance of choosing correct foils in memory tasks more broadly. Previous work has quantified how foil similarity at test drives memory performance arbitrarily lower for simple feature spaces, like color, when more similar foils are chosen (e.g., Schurgin et al., 2020). Here we show the same is true for complex and realistic stimuli, such as pictures of real-world objects, and offer a solution for how to quantify similarity in real-world object spaces using CNNs. Indeed, while the similarity structure of the CNN matches human similarity ratings closely, if anything it appeared to show more sensitivity to similarity between otherwise quite-dissimilar objects in a way that is important for memory performance, indicating that CNNs provide a particularly useful similarity measure in these circumstances. 
Importantly, we used this understanding of foil similarity to show that when stimulus pairs are not matched in foil similarity across stimulus sets this can result in false inferences about the capacity and nature of visual working memory. Specifically, we find that when choosing foils in a way that unfairly disadvantages objects relative to colors, the results of Quirk et al. (2020) and Li et al. (2020) - finding no visual working memory advantage for objects - can be replicated. However, when choosing foils in a way that is comparable across stimulus sets, objects are consistently better remembered than colors. Comparing performance across one dimension with maximally distinct foils (in the case of color) vs. another with randomly chosen crosscategory foils (in the case of objects: Li et al, 2020; Quirk et al, 2020) is incorrect: in the same way you would not compare memory for colors with foils being 180 degrees apart on the color wheel to memory for orientations with foils being 5 degrees apart and conclude colors are remembered better than orientations, this comparison does not provide a true measure of memory performance for objects vs. colors.

It is easy to overlook the necessity for using maximally-dissimilar foils from a stimulus set to assess working memory capacity. Indeed, these studies did try to make the real-world objects task comparable to the color task by choosing foils randomly from the Brady et al. (2008) stimulus set, thus to some extent limiting foils to be from a different object category (e.g., Quirk et al. 2020; Li et al. 2020). However, as we show in the current work, this does not maximize semantic or visual similarity of the objects, which are clearly important for visual working memory performance. In the current work, we used a novel method to maximize foil dissimilarity, using deep convolutional neural networks. By contrast, in our previous work, we maximized dissimilarity among object pairs solely by removing clearly similar target/foil pairs by hand (e.g. Brady et al. 2016). Is this difference critical? We believe it is not, and that any method of maximizing the similarity is sufficient to demonstrate the superiority of objects to colors in 
working memory. We originally found a significant benefit for objects relative to colors using the method of simply removing clearly similar target/foil pairs by hand, and we have recently shown that this object benefit result is replicable, as we found the same effect in 5 separate conditions in another paper which used the exact same stimuli set as Brady et al. (2016) (Brady \& Störmer, 2020). Thus, the critical reason why the Quirk et al. (2020) and Li et al. (2020) studies did not

find a performance advantage for objects does indeed seem to be that they made no attempt to maximize foil dissimilarity for objects, while explicitly maximizing dissimilarity for colors.

\section{What is a maximally-dissimilar foil?}

Some previous work has attempted to use 'cross-category' changes to make even larger changes than are possible within a given stimulus space and argue this provides an even more accurate measure of working memory capacity, as it even more fully maximizes the distinctiveness of the foils from the target item (e.g., Awh et al. 2007). For example, in their influential paper, Awh et al. (2007) showed a mix of 3D-cubes and Chinese characters and then at test had participants detect changes that could span categories: e.g., a cube could change to a character. They found this resulted in improved performance relative to within-category changes, and argued the ability to detect such changes more clearly reflected working memory capacity than the within-category changes.

There are two difficulties with using this approach to assess working memory capacity. The first is that such a measure does not assess capacity for a single stimulus set: instead, what is being measured is capacity for a new stimulus class consisting of both categories, e.g., capacity for 'character-cube' stimuli. In the context of such displays, the 'cross-category changes' are in fact just maximally distinct foils for the set of 'character-cubes'. Thus, the resulting capacity estimates are not estimates of how well either category can be remembered alone, but instead how well they can be remembered together. 
The second difficulty is that, contrary to the interpretation of Awh et al. (2007) about such changes, we have repeatedly found that introducing qualitative cross-category changes between two very dissimilar kinds of stimuli does more than just make foils more dissimilar: It also encourages people to use non-item-based ensemble encoding, effectively confounding memory capacity for objects with the more texture-based encoding strategies people use on such displays (Brady \& Alvarez, 2015; Schurgin \& Brady, 2019; Babic et al. 2019). In the same way that showing people a dozen colored circles and then, after a delay, probing them by asking if a mug was at a given location does not actually tap into memory limits for individual colors, qualitative changes to different categories of items also does not do so. Thus, overall, we believe the optimal way to measure working memory capacity for a given stimulus set is to maximize the distinctiveness of the foils within that set, but not to introduce qualitative differences in the stimuli as done by Awh et al. (2007).

\section{Even large, cross-category changes are not maximal}

Our results also reveal that even relatively large differences on the color wheel (e.g., categorically distinct colors, as used in Luck \& Vogel, 1997 and many follow-up papers), and large cross-category object changes (e.g., as used in Brady et al. 2008), do not result in the highest possible performance level. In the case of objects, this suggests that Brady et al. (2008), for example, underestimate the 'upper bound' of visual long-term memory performance. In the case of color, there are potentially even broader implications. We here show that performance is better with maximally distinct color foils than with random cross-category colors, consistent with other recent data on this issue (e.g., Schurgin et al. 2020), which show that even foils $>70$ degrees from the target on the color wheel do not give rise to maximal performance (compared to foils 180 degrees away). The continuous nature of this decrement in performance potentially raises difficulties for models of visual working memory which claim that items are either 
represented with relatively good precision, or else fundamentally unrepresented (i.e., forgotten; Zhang \& Luck, 2008; Adam et al. 2017). Instead, this data is more consistent with models that propose a continuous spreading of familiarity in the given feature space (Schurgin et al. 2020) and population-coding models that rely on shared neural representations to impose capacity constraints (Bays, 2015).

\section{Convolutional neural networks as models of memory confusability}

While only maximally dissimilar foils measure overall memory capacity non-arbitrarily for a given stimulus set, maximizing dissimilarity for objects is not straightforward. Here, rather than removing similar foils by hand, we instead offer a novel, objective solution to how similarity of real-world objects can be taken into account. In particular, we use a deep convolutional neural network trained to categorize visual objects to maximize dissimilarity. In recent years, CNNs have achieved impressively human-like object categorization performance, and these models have been argued to resemble the human visual system, with early and late layers of these networks tracking the human early and later processing pathways in the visual system, respectively (Khaligh-Razavi \& Kriegeskorte, 2014; Güçlü \& van Gerven, 2015; Cichy et al., 2016; Eickenberg et al., 2017). Because such networks are trained to do categorization, they are sensitive to both visual and semantic features (e.g., Jozwik et al. 2017; Peterson et al. 2018).

Here, we used CNNs as a measure of object similarity, and showed that the CNN similarity ratings match the pattern of similarity ratings by humans (Exp. 1), and the CNN-chosen foils significantly affected memory performance (Exp. 2 and 3), showing that they provide a useful metric for judging memory confusability. If anything, the small divergences of the human similarity and CNN seemed to favor the CNN: While maximally dissimilar objects and randomly chosen objects were judged only as slightly different in similarity by human observers - both 
being largely at floor — the CNNs predicted relatively larger similarity differences between these sets of stimuli. Interestingly, in Exp. 2 and Exp. 3 we find that memory performance differs quite a bit between these stimuli - i.e., we find a large increase in memory performance for maximally dissimilar objects relative to randomly chosen objects. At least qualitatively, this suggests memory performance more closely tracked the pattern of CNN similarity, although we cannot quantitatively dissociate them here as human similarity and CNN similarity were very strongly related.

Overall, we believe CNNs show significant promise for being integrated into the study of memory for real-world objects. Future research can examine the extent to which different CNN layers predict memory confusability (e.g., as a window in visual vs. semantic confusability), and how such metrics relate to different measures of human similarity.

\section{Other differences that may be relevant for colors vs. objects comparisons}

In the current paper we demonstrate the importance of foil similarity when comparing memory performance across different stimulus sets, and show how wrong inferences can be made about the nature of working memory capacity limits if foil dissimilarity is not matched. Is foil similarity the only factor important to consider when studying memory for real-world objects relative to colors?

In fact, while proper foils are a prerequisite to studying this issue, there are likely several other factors - for example, the way items are initially encoded — that play a critical role in eliciting benefits for real-world objects relative to colors (Brady \& Störmer, 2020). Specifically, when participants are encouraged to attend each item individually using focused attention processing each object in a deep way - real-world objects benefit, while color memory is impaired. By contrast, simple feature displays made out of only colors appear to benefit from 
fast and parallel encoding - presumably because of the use of non-item-based strategies such as ensemble processing or grouping (Brady \& Alvarez, 2015) — while real-world object memory is impaired in such circumstances (Brady \& Störmer, 2020). Thus, in addition to foil choice, there are other aspects of visual working memory that may lead to differences in how well real objects are encoded in memory and how this relates to colors.

In the current work, and in our previous work, we aim to achieve a level of deep processing, focused on individual items, by using long encoding times (e.g., Brady et al., 2016; Asp et al., 2019). However, in other recent work we have shown that while this does result in a benefit of objects relative to colors, this is far from the most effective manipulation in promoting a focused one-item-at-once encoding strategy, and a more effective manipulation is to actually show objects sequentially at encoding (one at a time; Brady \& Störmer, 2020). Overall, the evidence suggests that in addition to using proper foils, how the memory display is encoded initially is an important aspect of measuring memory capacity, and different strategies at encoding might lead to fundamentally different conclusions. Thus, variation in encoding strategy could be another reason why some other studies have failed to find an object benefit, in addition to using the incorrect foils at test (Li et al., 2020; Quirk et al., 2020).

\section{The role of meaning in visual working memory}

When target/foil similarity was matched, we found a robust and clear object benefit, replicating other work showing higher memory capacity for objects than colors (Brady et al., 2016; Brady \& Störmer, 2020). While comparing different stimulus sets in this way (e.g., color vs. objects) can provide insights on questions like the role of meaning or stimulus complexity in visual working memory, or the role of object complexity in memory performance, such comparisons are also difficult to interpret directly for conceptual reasons, in addition to the methodological issues that must be taken into account (as we have shown in the current work). 
For example, although we find objects are better remembered than colored circles, colored circles and real-world objects differ in many ways: objects are visually more complex; they are familiar; and they connect to categorical and semantic knowledge. For example, the increased visual complexity of real-world objects compared to colors means that objects, but not colors, differ on a number of dimensions, such as color, shape, luminance and orientation. Thus, the space of possible objects is far larger than that of simple features - which only differ in a singular dimension (e.g., color).

The current work does not seek to completely explain why objects are better remembered than colors. However, we believe the weight of the evidence suggests that it is the meaningfulness of objects that is critical, rather than these other factors (e.g., complexity). For example, previous studies have found that increased visual complexity — despite more complex objects having more dimensions they can differ on - actually result in lower working memory performance than simple features (e.g., Alvarez \& Cavanagh, 2004), suggesting that visual complexity alone is not the underlying factor behind enhanced working memory for real-world objects.

The hypothesis that meaningfulness is critical is supported by other work that has focused on stimuli that are nearly perfectly matched except in the critical dimension of interest. Indeed, many studies have used such methods to show significant benefits to visual working memory from familiarity and meaning (e.g., Alvarez \& Cavanagh, 2004; Asp et al. 2019; Jackson \& Raymond, 2008; Brady et al. 2009; Curby et al. 2009; Ngiam et al. 2019; O'Donnell, Clement, \& Brockmole, 2018; Sahar et al. 2020). For example, Asp et al. (2019) showed a benefit of meaning on visual working memory by using ambiguous stimuli that could either be recognized as meaningful (i.e., a face) or not, thus matching visual input while varying the meaningfulness of the stimuli, and found enhanced working memory performance and increased neural delay 
activity for nearly visually identical recognizable vs. non-recognizable stimuli. Results like these point to a clear role of meaningfulness in working memory and suggest that connections to knowledge, and not visual features - or the number of visual features - per se, improve working memory capacity.

Thus, overall we propose that the benefits for real-world objects relative to colors observed here, as in our other work (Brady et al., 2016; Brady \& Störmer, 2020), are in large part due to real-world objects being conceptually meaningful, and meaningful stimuli recruiting additional working memory resources.

\section{Conclusion}

The capacity of visual working and visual long-term memory play a critical role in theories of cognitive architecture and the relationship between memory and other cognitive systems. Here, we have shown that previous work in both visual long-term memory and visual working memory in particular has neglected to carefully consider one of the most important factors determining performance in a memory task, target/foil dissimilarity. Across 3 experiments, we showed that only by maximizing the dissimilarity of the target and foil in each stimulus set can we provide a fair basis for memory comparisons between stimuli, and we introduced a new way to pick such foils objectively for complex, meaningful real-world objects by using deep convolutional neural networks. This work thus demonstrates not only that working memory capacity is not fixed capacity but depends critically on the type of information that is remembered, but also offers a solution of how to compare memory performance and other cognitive systems across different stimulus sets on common ground. 


\section{Acknowledgements}

Support by NSF (BCS-1829434) to TFB/VSS.

\section{Open Practices Statement}

Materials, data and analysis code are available at https://osf.io/axyqs/

\section{References}

Adam, K. C., Vogel, E. K., \& Awh, E. (2017). Clear evidence for item limits in visual working memory. Cognitive psychology, 97, 79-97.

Alvarez, G. A., \& Cavanagh, P. (2004). The capacity of visual short-term memory is set both by visual information load and by number of objects. Psychological science, 15(2), 106-111.

Asp, I., Störmer, V. S., \& Brady, T. (2019). Greater visual working memory capacity for visuallymatched stimuli when they are recognized as meaningful. PsyArxiv, https://doi.org/10.31234/osf.io/r6njf

Awh, E., Barton, B., \& Vogel, E. K. (2007). Visual working memory represents a fixed number of items regardless of complexity. Psychological science, 18(7), 622-628.

Baddeley, A. (2012). Working memory: Theories, models, and controversies. Annual review of psychology, 63, 1-29.

Babic, Z., Schurgin, M.W., and Brady, T.F. (2019). Is short-term storage correlated with fluid intelligence? Strategy use explains the apparent relationship between 'number of remembered items' and fluid intelligence. PsyArXiv, https://doi.org/10.31234/osf.io/83ch4

Bays, P. M. (2015). Spikes not slots: noise in neural populations limits working memory. Trends in cognitive sciences, 19(8), 431-438.

Brady, T. F., \& Alvarez, G. A. (2015). No evidence for a fixed object limit in working memory: Spatial ensemble representations inflate estimates of working memory capacity for complex objects. Journal of Experimental Psychology: Learning, Memory, and Cognition, 41(3), 921.

Brady, T. F., Konkle, T., Alvarez, G. A., \& Oliva, A. (2008). Visual long-term memory has a massive storage capacity for object details. Proceedings of the National Academy of Sciences, 105(38), 14325-14329. 
Brady, T. F., Konkle, T., \& Alvarez, G. A. (2009). Compression in visual working memory: Using statistical regularities to form more efficient memory representations. Journal of Experimental Psychology: General, 138(4), 487.

Brady, T.F. \& Störmer, V.S (2020). The role of meaning in visual working memory: Real-world objects, but not simple features, benefit from deeper processing. PsyArxiv.

Brady, T. F., Störmer, V. S., \& Alvarez, G. A. (2016). Working memory is not fixed-capacity: More active storage capacity for real-world objects than for simple stimuli. Proceedings of the National Academy of Sciences, 113(27), 7459-7464.

Brady, T.F. \& Störmer, V.S (2020). Comparing memory capacity across stimuli requires maximally dissimilar foils: Using deep convolutional neural networks to understand visual working memory capacity for real-world objects. Retrieved from https://osf.io/axyqs/

Cowan, N. (2001). The magical number 4 in short-term memory: A reconsideration of mental storage capacity. Behavioral and Brain Sciences, 24(1), 87-114.

Curby, K. M., Glazek, K., \& Gauthier, I. (2009). A visual short-term memory advantage for objects of expertise. Journal of Experimental Psychology: Human Perception and Performance, 35(1), 94.

Güçlü, U., \& van Gerven, M. A. (2015). Deep neural networks reveal a gradient in the complexity of neural representations across the ventral stream. Journal of Neuroscience, 35(27), 10005-10014.

He, K., Zhang, X., Ren, S., \& Sun, J. (2016, October). Identity mappings in deep residual networks. In European conference on computer vision (pp. 630-645). Springer, Cham.

Frank, D., Gray, O., \& Montaldi, D. (2020). SOLID-Similar object and lure image database. Behavior Research Methods, 52(1), 151-161.

Jackson, M. C., \& Raymond, J. E. (2008). Familiarity enhances visual working memory for faces. Journal of Experimental Psychology: Human Perception and Performance, 34(3), 556.

Jozwik, K. M., Kriegeskorte, N., Storrs, K. R., \& Mur, M. (2017). Deep convolutional neural networks outperform feature-based but not categorical models in explaining object similarity judgments. Frontiers in psychology, 8, 1726.

Kar, K., Kubilius, J., Schmidt, K., Issa, E. B., \& DiCarlo, J. J. (2019). Evidence that recurrent circuits are critical to the ventral stream's execution of core object recognition behavior. Nature neuroscience, 22(6), 974-983. 
Khaligh-Razavi, S. M., \& Kriegeskorte, N. (2014). Deep supervised, but not unsupervised, models may explain IT cortical representation. PLoS computational biology, 10(11), e1003915.

Kietzmann, T. C., McClure, P., \& Kriegeskorte, N. (2018). Deep neural networks in computational neuroscience. BioRxiv, 133504.

Kubilius, J., Schrimpf, M., Nayebi, A., Bear, D., Yamins, D. L., \& DiCarlo, J. J. (2018). Cornet: Modeling the neural mechanisms of core object recognition. BioRxiv, 408385.

Landauer, T. K. (1986). How much do people remember? Some estimates of the quantity of learned information in long-term memory. Cognitive science, 10(4), 477-493.

Li, X., Xiong, Z., Theeuwes, J., \& Wang, B. (2020). Visual memory benefits from prolonged encoding time regardless of stimulus type. Journal of Experimental psychology. Learning, Memory, and Cognition.

Lindsay, G. (2020). Convolutional neural networks as a model of the visual system: past, present, and future. Journal of Cognitive Neuroscience, 1-15.;

Luck, S. J., \& Vogel, E. K. (1997). The capacity of visual working memory for features and conjunctions. Nature, 390(6657), 279-281.

Luck, S. J., \& Vogel, E. K. (2013). Visual working memory capacity: from psychophysics and neurobiology to individual differences. Trends in cognitive sciences, 17(8), 391-400.

Ma, W. J., Husain, M., \& Bays, P. M. (2014). Changing concepts of working memory. Nature neuroscience, 17(3), 347.

Mate, J., \& Baqués, J. (2009). Short article: Visual similarity at encoding and retrieval in an item recognition task. Quarterly Journal of Experimental Psychology, 62(7), 1277-1284.

Ngiam, W. X., Khaw, K. L., Holcombe, A. O., \& Goodbourn, P. T. (2019). Visual working memory for letters varies with familiarity but not complexity. Journal of Experimental Psychology: Learning, Memory, and Cognition, 45(10), 1761.

O'Donnell, R. E., Clement, A., \& Brockmole, J. R. (2018). Semantic and functional relationships among objects increase the capacity of visual working memory. Journal of Experimental Psychology: Learning, Memory, and Cognition, 44(7), 1151.

Palmeri, T. J., \& Tarr, M. (2008). Visual object perception and long-term memory. Visual memory, 163-207. 
Peterson, J. C., Abbott, J. T., \& Griffiths, T. L. (2018). Evaluating (and improving) the correspondence between deep neural networks and human representations. Cognitive science, 42(8), 2648-2669.

Quirk, C., Adam, K.C.S., \& Vogel, E.K. (2020). No evidence for an object working memory capacity benefit with extended viewing time. PsyArxiv, https://osf.io/pzy5q/

Russakovsky, O., Deng, J., Su, H., Krause, J., Satheesh, S., Ma, S., ... \& Berg, A. C. (2015). Imagenet large scale visual recognition challenge. International journal of computer vision, 115(3), 211-252.

Sahar, T., Sidi, Y., \& Makovski, T. (2020). A Metacognitive Perspective of Visual Working Memory With Rich Complex Objects. Frontiers in Psychology, 11, 179.

Schurgin, M. W., \& Brady, T. F. (2019). When "capacity" changes with set size: Ensemble representations support the detection of across-category changes in visual working memory. Journal of vision, 19(5), 3-3.

Schurgin, M. W., Wixted, J. T., \& Brady, T. F. (2018). Psychophysical scaling reveals a unified theory of visual memory strength. BioRxiv, 325472.

Simonyan, K., \& Zisserman, A. (2014). Very deep convolutional networks for large-scale image recognition. arXiv, 1409.1556.

Standing, L. (1973). Learning 10000 pictures. The Quarterly journal of experimental psychology, 25(2), 207-222.

Storrs, K. R., Kietzmann, T. C., Walther, A., Mehrer, J., \& Kriegeskorte, N. (2020). Diverse deep neural networks all predict human IT well, after training and fitting. bioRxiv.

Suchow, J. W., Brady, T. F., Fougnie, D., \& Alvarez, G. A. (2013). Modeling visual working memory with the MemToolbox. Journal of vision, 13(10), 9-9.

Wilken, P., \& Ma, W. J. (2004). A detection theory account of change detection. Journal of vision, 4(12), 11-11.

Xu, Y., \& Vaziri-Pashkam, M. (2020). Limited correspondence in visual representation between the human brain and convolutional neural networks. BioRxiv.

Yamins, D. L., \& DiCarlo, J. J. (2016). Using goal-driven deep learning models to understand sensory cortex. Nature neuroscience, 19(3), 356-365. 
Yamins, D. L., Hong, H., Cadieu, C. F., Solomon, E. A., Seibert, D., \& DiCarlo, J. J. (2014). Performance-optimized hierarchical models predict neural responses in higher visual cortex. Proceedings of the National Academy of Sciences, 111(23), 8619-8624.

Zhang, W., \& Luck, S. J. (2008). Discrete fixed-resolution representations in visual working memory. Nature, 453(7192), 233-235. 